\title{
T-SP1: a novel serine protease-like protein predominantly expressed in testis
}

\author{
Peter Neth ${ }^{1, a, *}$, Birgit Profanter ${ }^{1, a}$, Claudia \\ Geissler ${ }^{1}$, Dorit K. Nägler ${ }^{1}$, Andreas Nerlich², \\ Christian P. Sommerhoff' and Marianne \\ Jochum ${ }^{1}$ \\ ${ }^{1}$ Division of Clinical Chemistry and Clinical \\ Biochemistry, Department of Surgery, Ludwig \\ Maximilians University, D-80336 Munich, Germany \\ ${ }^{2}$ Department of Pathology, Academic Hospital \\ Munich-Bogenhausen, D-81925 Munich, Germany \\ ${ }^{*}$ Corresponding author \\ e-mail: Peter.Neth@med.uni-muenchen.de
}

\begin{abstract}
Here, we describe a novel member in the group of membrane-anchored chymotrypsin (S1)-like serine proteases, namely testis serine protease 1 (T-SP1), as it is principally expressed in testis tissue. The human T-SP1 gene encompasses $28.7 \mathrm{~kb}$ on the short arm of chromosome 8 and consists of seven exons. Rapid amplification of cDNA ends (RACE) experiments revealed that due to alternative splicing three different variants (T-SP1/1, $-2,-3)$ are detectable in testis tissue displaying pronounced heterogeneity at their $3^{\prime}$-end. T-SP $1 / 1$ consists of an 18 amino acid signal peptide and of a 49 amino acid propeptide. The following domain with the catalytic triad of $\mathrm{His}^{108}$, Asp ${ }^{156}$, and $\mathrm{Ser}^{250}$ shares sequence identities of $42 \%$ and $40 \%$ with the blood coagulation factor $\mathrm{XI}$ and plasma kallikrein, respectively. Only T-SP1/1 contains a hydrophobic part at the C-terminus, which provides the basis for cell membrane anchoring. Using a newly generated polyclonal anti-T-SP1 antibody, expression of the T-SP1 protein was found in the Leydig and Sertoli cells of the testis and in the epithelial cells of the ductuli efferentes. Notably, T-SP1 protein was also detectable in prostate cancer and in some ovarian cancer tissues, indicating tumor-related synthesis of T-SP1 beyond testis tissue.
\end{abstract}

Keywords: alternative splicing; catalytic triad; leydig cells; ovarian cancer; prostate cancer; sertoli cells; testis serine protease 1 (T-SP1).

\section{Introduction}

Serine proteases are found in a wide range of tissues and biological fluids, playing crucial roles in the development and maintenance of the organism, including fertilization, immune responses, wound healing, blood coagulation, and digestion. Furthermore, these proteases contribute

a These authors contributed equally to this work. to many diseases and are particularly involved in multiple organ failure due to sepsis and trauma (Jochum et al., 1994; Waydhas et al., 1996), as well as tumor progression (Johnsen et al., 1998; Foekens et al., 2003; Reuning et al., 2003; Yousef and Diamandis, 2003).

A new group of the chymotrypsin (S1)-like serine proteases was recently identified that contain domains which anchor them to the plasma membrane (NetzelArnett et al., 2003). A subgroup of the membrane-bound serine proteases which is anchored via a GPI-linkage includes prostasin (Yu et al., 1995; Chen et al., 2001) and testisin (Hooper et al., 1999, 2000), two proteases with an expression pattern being restricted to the male genital tract. While prostasin is mainly detectable in the prostate gland, testisin is specifically expressed by testicular germ cells during the premeiotic stage of spermatogenesis (Hooper et al., 1999). Structural features and specific expression patterns of testisin suggest that it regulates proteolytic events associated with testicular germ cell development (Hooper et al., 1999, 2000). Further serine proteases which seem to play a (patho)physiological role, especially in the male genital tract or in cancer progression, belong to the human kallikrein-related peptidases (Lundwall et al., 2006; Paliouras and Diamandis, 2006; Whitbread et al., 2006; Lundwall and Brattsand, 2008), encoded by the kallikrein gene locus which comprises 15 serine protease genes (Yousef et al., 2000; Clements et al., 2001) with prostate-specific antigen (KLK3) as one of the best known representatives (Yousef and Diamandis, 2003; Lilja et al., 2008).

Due to our interest in kallikreins (especially plasma kallikrein) (Neth et al., 2001, 2005; Fink et al., 2007), we searched for transcripts encoding related enzymes in the human genome. Our search in the NCBI database led to a new family member of serine proteases which was firstly named 'Similar to Plasma Prekallikrein Precursor' or GPI-SP1 (Netzel-Arnett et al., 2003).

mRNA expression profiling revealed that this gene is predominantly transcribed in testis. We therefore termed this new protein as T-SP1 (testis serine protease 1). In addition to the database protein sequences of T-SP1/1 and T-SP1/2, a third splice variant was detected, which we termed T-SP1/3. Only T-SP1/1 encodes a chymotrypsin-like serine protease, which also contains a hydrophobic $\mathrm{C}$-terminal sequence, suggesting a membrane-bound localization. Immunohistochemical studies with a newly generated polyclonal antibody revealed T-SP1 expression in Leydig and Sertoli cells of the testis, in the epithelial cells of the ductuli efferentes, and interestingly also in prostate cancer, as well as ovarian cancer tissues.

\section{Results}

Identification, cloning, and bioinformatics on T-SP1

Tissue-specific expression of human T-SP1 mRNA Following the bioinformatics studies on T-SP1, we 
designed primers for the predicted coding sequence of human T-SP1 according to the GenBank sequence (NM_198464, Homo sapiens tryptophan/serine protease). Tissue distribution of T-SP1 expression was analyzed by quantitative PCR using 16 different human tissue cDNAs. T-SP1-mRNA was expressed in low amounts exclusively in testis, displaying a T-SP1/GAPDH ratio of $18.9 \times 10^{-4}$ (Figure 1). NCBI unigene analysis revealed a total of 17 EST entries for T-SP1, 11 for testis tissue, 5 for brain tissue, and 1 for an uncharacterized tissue. Interestingly, neither the primer pair designed for qPCR nor that for the amplification of the entire coding region detected T-SP1 expression in human brain tissue.

Cloning, transcript analysis, and exon-intron structure of the human T-SP1 gene To identify the transcriptional start site(s) of T-SP1, we used poly $(A)^{+}$mRNA of human testis for RLM-RACE (RNA ligase-mediated rapid amplification of cDNA ends). 5'-RLM-RACE ensures that only full-length $5^{\prime}$-ends of mRNA molecules with an intact cap structure are selectively reverse transcribed and amplified by nested PCR. By analysis of 18 clones, nine different transcriptional start sites were detected in close proximity, forming two clusters with 11 and $15 \mathrm{bp}$ in length (Figure 2A). The most frequently detected transcription start sites were at positions -97 ( 6 clones) and at -54 ( 3 clones). The resulting 5 '-untranslated regions vary from 45 to $105 \mathrm{bp}$ in length. No further upstream exon was detected.

In a next step the $3^{\prime}$-end of the T-SP1 transcript was examined. Thereby, $3^{\prime}$-RACE analysis revealed a marked heterogeneity at the $3^{\prime}$-tail resulting in T-SP1 mRNAmolecules of different length. In total, we detected three different splice variants encoding mRNA molecules with open reading frames of $1059 \mathrm{bp}, 831 \mathrm{bp}$, and $912 \mathrm{bp}$ (Figure 2B,C). The predicted preproT-SP1 proteins comprise 352,276 , and 303 amino acids with a molecular mass of $38.9 \mathrm{kDa}, 30.7 \mathrm{kDa}$, and $33.6 \mathrm{kDa}$, respectively. Comparison of the mRNA sequences of all three variants with the NCBI database revealed that entry NM_198464 is identical to variant T-SP1/1, and T-SP1/2 is represented by the mRNA BC033497, while T-SP1/3 is a newly

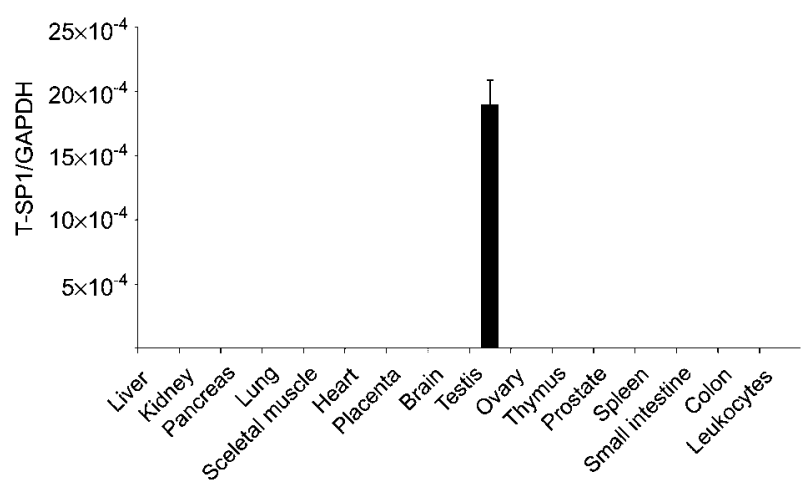

Figure 1 Analysis of human T-SP1 expression in human tissues.

Quantitative RT-PCR was carried out on 16 different human tissues using $1 \mathrm{ng}$ cDNA. For that, a primer pair was used which allows detection of all three T-SP1 splice variants simultaneously. GAPDH transcripts were quantified in the same cDNAs which served as normalization control. detected form. For the quantification of the different splice variants, three separate, isoform-specific primer pairs were designed. The results showed that the main expression concerns T-SP1/1, whereas T-SP1/2 and TSP1/3 are transcribed in much lower amounts (T-SP1/1: 99.87\%, T-SP1/2: $0.13 \%$ and T-SP1/3: $\left.<4 \times 10^{-4} \%\right)$. For $\mathrm{T}-\mathrm{SP} 1 / 1$, the cDNA sequence with the corresponding amino acid sequence is shown in Figure $2 \mathrm{~B}$.

The genomic structure of the T-SP1 gene (Figure 2A) was deduced by comparison of the three T-SP1 transcripts with the genomic T-SP1 sequence on chromosome 8 (8p23.1, range 2858342-2887039) in combination with the $5^{\prime}-$ and $3^{\prime}$-RACE results. The results revealed that T-SP1/1 is derived from exons $1,2,3,4$, and 6 , whereas T-SP1/2 is encoded by exons $1,2,3,4$, and 7 , and T-SP1/3 variant comprises exons 1, 2, 3, 4, 5 , and 7 (Figure $2 \mathrm{C}$ ), indicating that the human T-SP1 gene includes seven exons spanning approximately $28.7 \mathrm{kbp}$ on chromosome 8p23.1 (Figure 2A). The lengths of the exons (1-7) range from 81 to $349 \mathrm{bp}$ and those of the introns are between 1360 and $15149 \mathrm{bp}$ (Figure 2A). Alternative splicing in the region of intron 4 up to exon 7 causes excision of this sequence in T-SP1/2, whereas the sequence of intron 5 up to exon 7 is removed in T-SP1/3 (Figure $2 \mathrm{C}$ ).

\section{Protein structure of human T-SP1 and sequence iden-} tity with other human serine proteases The predicted preproproteins of all three T-SP1 variants start with a typical 18-amino acid signal peptide for a nascent secretory protein. According to bioinformatic analysis, the propeptide of the zymogen begins with Gly ${ }^{19}$, the propeptide comprises 49 residues and ends with $\operatorname{Arg}^{67}$ (Figure 2B).

In contrast to the common $\mathrm{N}$-terminal part of the catalytic domain, the C-terminal part is heterogeneous among the three different T-SP1 splice variants. T-SP1/1 comprises a full-length serine protease domain of 228 amino acids, where all essential amino acids around the catalytic triad $\left(\mathrm{His}^{108}, \mathrm{Asp}^{156}\right.$, and $\mathrm{Ser}^{250}$ ) are highly conserved (Figure 2B). Based on alignment and structural data of other serine proteases (McMullen et al., 1991a,b) in humans, T-SP1/1 disulfide bonds may be formed between Cys ${ }^{53} /$ Cys $^{176}$, Cys $^{93} /$ Cys $^{109}$, Cys ${ }^{189} /$ Cys $^{256}$, Cys ${ }^{222} /$ $\mathrm{Cys}^{235}$ and Cys ${ }^{246} /$ Cys $^{276}$ (Figure $3 \mathrm{~A}$ ). The first of these cysteine pairs is predicted to link the pro- and catalytic domain, whereas the remainder are likely to form disulfide bonds within the catalytic domain. Interestingly, a presumably unpaired Cys exists at residue 340 close to the C-terminus. Hydropathy plot analysis of T-SP1/1 revealed that this free Cys is located within a highly hydrophobic sequence of 17 residues (WLLLCPLSHVLFRAILY), which may function as an anchor to the plasma membrane (Figures 2B and 3A). T-SP1 splice variants 2 and 3 are identical to isoform 1 up to the lysine residue at position 247 (Figure 2B). In the following sequence part, the highly conserved GDSGGP motif of chymotrypsin-like serine proteases which is also found in T-SP1/1 is absent in T-SP1/2 and T-SP1/3, respectively (data not shown). Thus, with respect to the serine residue of the catalytic triad of T-SP1/1 no conserved equivalent amino 
A

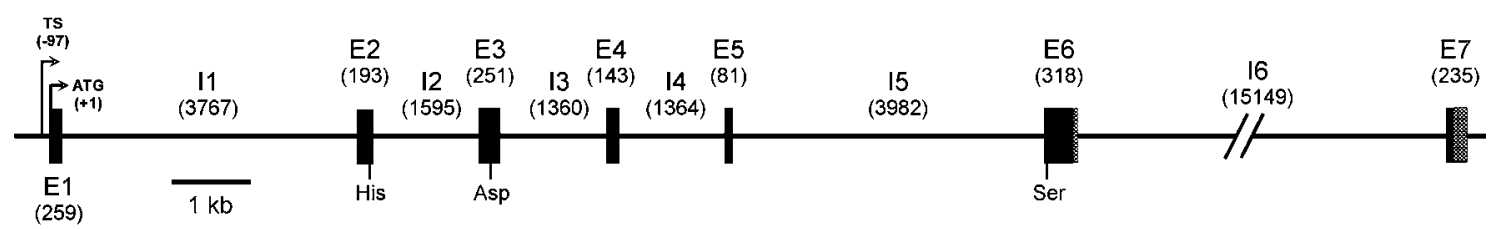

B

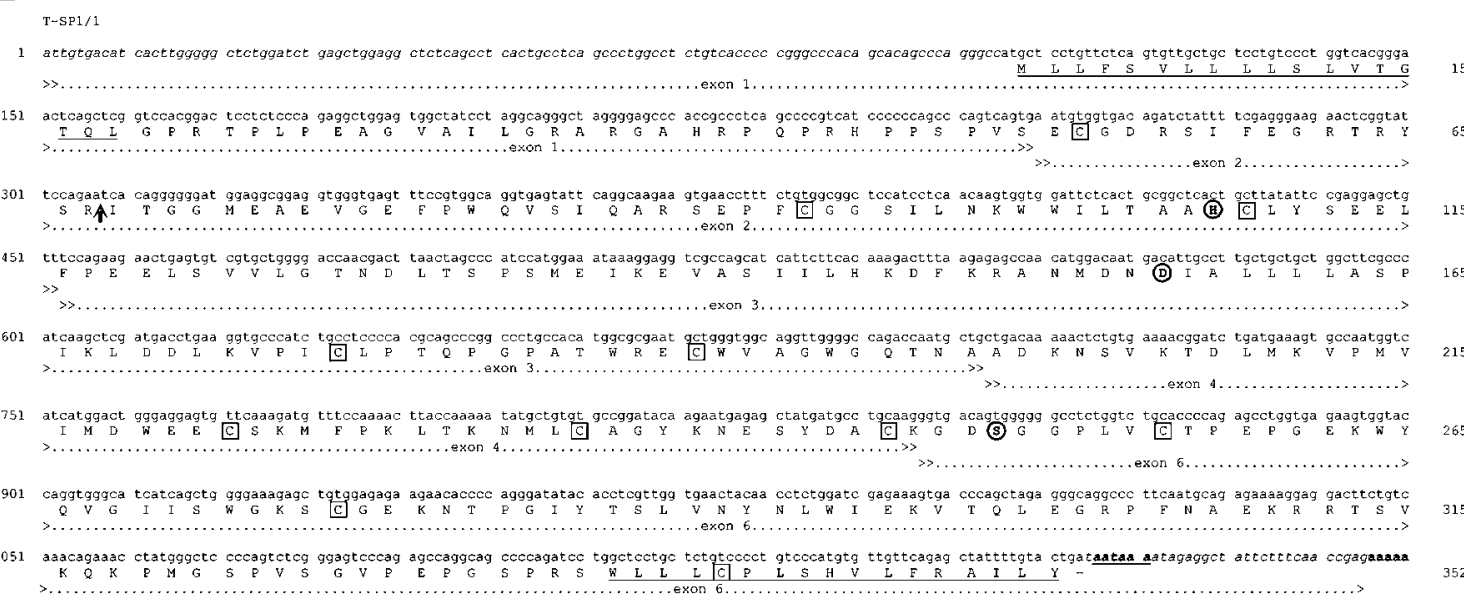

C

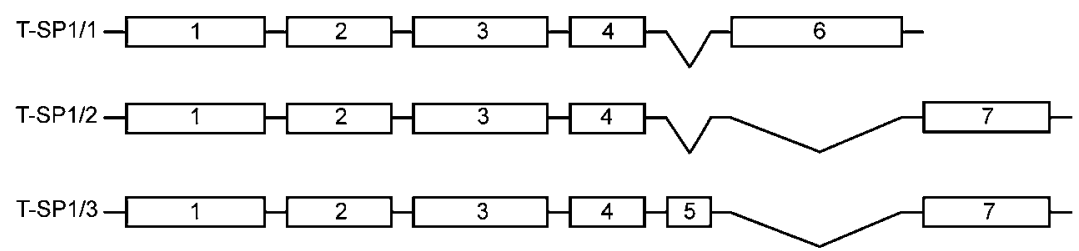

Figure 2 Genomic organization, nucleotide and amino acid sequence, and exon distribution of the human T-SP1 gene.

(A) Genomic organization of the human T-SP1 gene. The human T-SP1 gene consists of seven exons and six introns and encompasses $28.7 \mathrm{~kb}$ on human chromosome 8. Black boxes represent the seven exons (E1-E7) and the six introns are indicated by the black line (11-16). Exon and intron lengths are indicated in parentheses. 5'-RLM-RACE revealed transcription start sites at positions -105 , $-97,-94,-60,-54,-53,-51,-46$, and -45 . Only the transcription start site at position -97 is indicated (TS), as this start site was the preferred position for the initiation of transcription. The $3^{\prime}$-untranslated regions are designated by a hatched box. His, Asp, and Ser refer to the catalytic triad amino acids in exons 2, 3, and 6. (B) Nucleotide and amino acid sequences of human T-SP1/1. Nucleotides are numbered on the left and amino acids on the right site. Residues of the catalytic triad are highlighted in bold circles. The putative activation site (ITGG) is indicated by an arrow and cysteines are marked with boxes. The N-terminal signal sequence (18 aa) is followed by the putative propeptide with 49 amino acids. The C-terminal hydrophobic region (17 aa) is underlined. The polyadenylation signal is underlined in bold. The $5^{\prime}$-untranslated region (105 bp) is indicated in cursive. The $3^{\prime}$-untranslated region from T-SP1/1 (31 bp) is also shown in cursive letters. The poly(A) tail starts at position 1196. T-SP1 encodes for an mRNA molecule which comprise an open reading frame of 1059 bp giving rise to a protein with 352 amino acids. (C) Exon distribution of SP1 splice variants. T-SP1/1 is encoded by exons 1-4 and 6, whereas T-SP1/2 is composed of exons $1-4$ and 7 . T-SP $1 / 3$ consists of exons $1-5$ and 7 . In T-SP1/1 the active site Ser ${ }^{250}$ is encoded by exon 6 . Due to the absence of exon 6 in T-SP $1 / 2$ and T-SP $1 / 3$, this highly conserved region at $\mathrm{Ser}^{250}$ is absent in these splice variants.

acid sequence in the two other variants exists. Furthermore, the hydrophobic C-terminus is absent in the TSP1/2 and T-SP1/3.

To identify the nearest related protease members of $\mathrm{T}-\mathrm{SP} 1 / 1$, alignment studies were performed. The predicted amino acid sequence of the catalytic domain of T-SP1/1 shares $42 \%$ and $40 \%$ identity with human coagulation factor XI and plasma kallikrein (Figure 3B). A slightly lower sequence identity was identified with the male genital tract proteases testisin (36\%), prostasin $(35 \%)$, acrosin $(35 \%)$, and leydin (33\%). With respect to kallikrein-related peptidases, T-SP1/1 protein shares reasonable sequence identity with the human KLK2 (32\%) and KLK13 (37\%). In addition, T-SP1/1 shows 34\% sequence identity with trypsin (Figure $3 \mathrm{~B}$ ).

\section{Recombinant expression, antibody generation, and immunolocalization of T-SP1}

Recombinant expression of proT-SP1 variants in Escherichia coli For the production of specific antibodies, we generated the antigens by recombinant expression of proT-SP1/1, proT-SP1/2, and proT-SP1/3 in $E$. coli. After purification by $\mathrm{Ni}$-affinity chromatography the recombinant proteins were analyzed by SDS-PAGE (data not shown). The protein yields after purification ranged between $20 \mu \mathrm{g}$ (proT-SP1/1), $25 \mu \mathrm{g}$ (proT-SP1/ 2), and $50 \mu \mathrm{g}$ (proT-SP1/3) per liter $E$. coli culture.

Antibody generation Taking the relatively higher expression rate of T-SP1/3 in E. coli into account, an anti- 
A

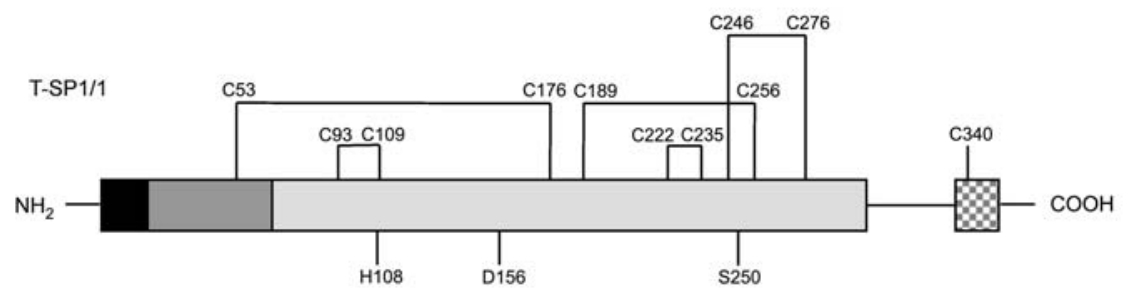

B

T-SP1 FAKTOR XI PPK PROSTASIN TESTISIN ACROSIN LEYDIN KLK2

KLK13

TRYPSIN CONSENSUS

T-SP1 FAKTOR XI PPK PROSTASIN TESTISIN ACROSIN LEYDIN

KLK2

KLK13

TRYPSIN CONSENSUS

T-SP1 FAKTOR XI PPK PROSTASIN TESTISIN ACROSIN LEYDIN

KLK2

KLK13

TRYPSIN

CONSENSUS

T-SP1 FAKTOR XI PPK PROSTASIN TESTISIN ACROSIN LEYDIN KLK2

KLK13

TRYPSIN CONSENSUS

T-SP1 FAKTOR XI PPK PROSTASIN TESTISIN ACROSIN LEYDIN KLK2

KLK13

TRYPSIN

CONSENSUS
68 ITGGMEAEVGEFPWQVSIQA---R---SEP-----F--CGGSILNKWWILTAAHCL--YSEELFP--EEL 388 IVGGTASVRGEWPWQVTLHT---T---SPTQRH--L--CGGSIIGNQWILTAAHCF--YGVES-P--KII 391 IVGGTNSSWGEWPWQVSLQV---KLTAQRH-----L--CGGSLIGHQWVLTAAHCF--DGLPLQDVWRIY

45 ITGGSSAVAGQWPWQVSITY---E---GVH-----V--CGGSLVSEQWVLSAAHCF--PSEH-HK--EAY 42 IVGGEDAELGRWPWQGSLRL---W---DSH-----V--CGVSLLSHRWALTAAHCFETYSDLSDP--SGW 43 IVGGKAAQHGAWPWMVSLQI FTYN---SHR-----YHTCGGSLLNSRWVLTAAHCF--VGKNNV---HDW 17 IIGGKNSLRGGWPWQVSLRL---K---SSHGDGRLL--CGVTLLSSCWVLTAAHCF--KRYGNST--RSY 25 IVGGWECEKHSQPWQVAVYS---H---GWA-----H--CGGVLVHPQWVLTAAHCL--------K--KNS 36 LPGGYTCFPHSQPWQAALLV---Q---GRL-----L--CGGVLVHPKWVLTAAHCL--------K--EGI 24 IVGGYNCEENSVPYQVSLNS-------GYH-----F--CGGSLINEQWVVSAGHC--------YK--SRI GG CG W A HC

121 SVVLGTNDL--T--------S-PSMEIK--E-------VA-----------SIILHKDFKR-A----NM 443 RVYSGILN-------------QSEIK--EDTSFFG-VQ-----------EIIIHDQYKM-A----ES 449 SGILNLSDI--TKD------T-PFSQIK--E-_-_-_97 EVKLGAHQL--D-------S-YSEDAKVST------LLK---------DIIPHPSYLQ-E----GS 97 MVQFG--QL--T--------SMPSEWSL--QAYYTRYFVS----------NIYLSPRYL--G----NS 100 RLVEGAKEI--TYGNNKPVKA-PVQE-R--Y------VE----------KIIIHEKYNS-A----TE 75 AVRVGDYHT--L--------V-P-VEFE--EEIG----VQ-----------QIVIHREYRP-D----RS 72 QVWLGRHNL--F--------E-PEDTGQ--R------VPVSHSFPHPLYNMSLLKHQSLRPDE----DS 83 KVYLGKHALGRV-------E-AGEQVR--E------VV-------HSIPHPEYRR-SPTHLNH 70 QVRLGEHNI--EVL------E-GNEQFI--N------AA--------KIIRHPQYDR-K----TL

154 DNDIALLLLASP----IKLDDL--KVPICLPTQPG--P-AT-WR-E--------CWVAGWG--OTNAADK 478 GYDIALLKLETT----VNYTDS--QRPICLPSKGD--RNVI-YT-D--------CWVTGWG--YRKLRDK 481 NHDIALIKLQAP----LNYTEF--QKPICLPSKGD--T-STIYT-N--------CWVTGWG--FSK--EK 132 QGDIALLQLSRP----ITFSRY--IRPICLPAANA--SFPN-GL-H--------CTVTGWG--HVAPSVS 135 PYDIALVKLSAP----VTYTKH--IQPICLQASTF--E-FE-NRTD--------CWVTGWG--YIKEDEA 140 GNDIALVEITPP----ISCGRF--IGPGCLPHLKAGLP-RG-SO-S-------CWVAGWGYIEEKAPRP 110 DYDIALVRLQGPEEQCARESSH--VLPACLP-------L-WR-ERPQKTASNCYITGWG--DTGRAYS 118 SHDLMLLRLSEP----AKITDV--VKVLGLPTQE---P-AL-GT-T-------CYASGWG--SIEPEEF 122 DHDIMLLELQSP----VQLTGYIQTLPLSHNNRLT--P-GT----T-------CRVSGWG--TTTSPQV 105 NNDIMLIKLSSR----AVINAR--VSTISLPTAP---P-AT-GT-K--------CLISGWG--NT-ASSG L

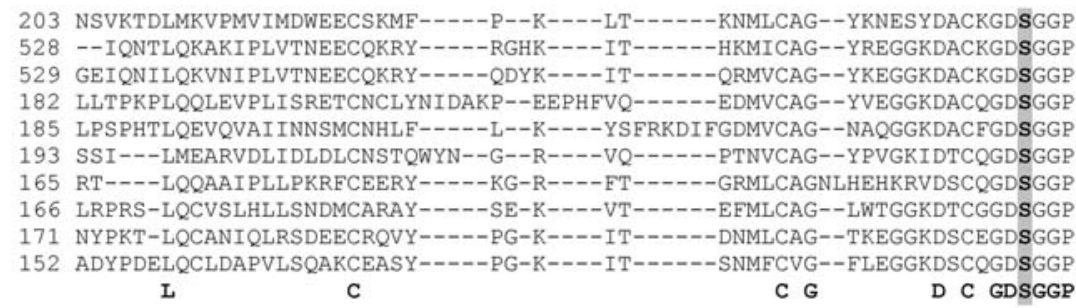

254 LVCTPEPGEKWYQVGIISWG-K-SCGEKNTPGIYTSLVNYNLWI 579 LSC--KHNEVWHLVGITSWG-E-GCAORERPGVYTNVVEYVDWI 582 LVC--KHNGMWRLVGITSWG-E-GCARREQPGVYTKVAEYMDWI 242 LSC-PVEG-LWYLTGIVSWG-D-ACGARNRPGVYTLASSYASWI 242 LAC--NKNGLWYQIGVVSWG-V-GCGRPNRPGVYTNISHHFEWI 244 LMCKDSKESAYVVVGITSWG-V-GCARAKRPGIYTATWPYLNWI 215 LMC-ERPGESWAALGVTSWG-Y-GCGVKDSPGVYTKVSAFVPWI 217 LVC------NGVLQGITSWGPE-PCALPEKPAVYTKVVHYRKWI 222 LVCNRT-----LYGIVSWG-DFPCGQPDRPGVYTRVSRYVLWI 204 VVCNGQ------LQGVVSWG-D-GCAQKNKPGVYTKVYNYVKWI SWG C PG YT

Figure 3 Predicted structure of human T-SP1 (A) and alignment of T-SP1/1 with related human serine proteases (B).

(A) The cDNA sequence predicts that T-SP1/1 is translated initially as a single chain, 352 amino acid precursor with a signal peptide (black), propeptide (dark gray), a catalytic domain (light gray), and a hydrophobic stretch (patterned) at the C-terminus. We predict that the signal peptide is removed co-translationally in the endoplasmic reticulum, leaving a proenzyme which is activated subsequently by hydrolysis of the propeptide segment at $\mathrm{Arg}^{67}$. This results in a 285-residue catalytic domain (heavy chain) through a disulfide linkage involving Cys ${ }^{53}$ and Cys ${ }^{176}$. The location of other Cys-Cys pairs is shown by analogy to the Cys-pairings in human plasma kallikrein. Also shown are positions of the catalytic triad residues found in all active serine proteases (His ${ }^{108}$, Asp ${ }^{156}$, and $\mathrm{Se}^{250}$, corresponding to His ${ }^{57}$, Asp ${ }^{102}$, and Ser ${ }^{195}$ using standard chymotrypsinogen numbering). (B) T-SP1/1 shows sequence identity to human factor XI (42\%), to human plasma kallikrein (PPK) (40\%), and to human prostasin (38\%). Sequence identity was found for the serine proteases testisin (36\%) and acrosin (35\%). For leydin, the sequence identity was calculated at $33 \%$. T-SP1/1 contains the catalytic triad with $\mathrm{His}^{108}$, Asp ${ }^{156}$, and $\mathrm{Ser}^{250}$ (in bold letters and gray boxes). T-SP1/1 protein also shares sequence similarity with kallikrein-related peptidase 2 (KLK2) (32\%), and KLK 13 (37\%). 
T-SP1 polyclonal antibody was generated by immunizing rabbits with recombinant T-SP1/3. Owing to the substantial amino acid sequence identity of $82 \%$ between variant T-SP1/1 and T-SP1/3 one could expect effective crossreactivity of T-SP1/1 with anti-T-SP1/3 antibodies. The generated polyclonal antibody detects the purified variants T-SP1/1, T-SP1/2, and T-SP1/3 in Western blot experiments which were further confirmed by using the anti-penta-His antibody for identification of the variants via their C-terminal His-tag (data not shown).

\section{Immunohistochemical detection of T-SP1 in testis} and cancer tissues Applying the polyclonal anti-TSP1 antibody for immunofluorescence detection, we localized T-SP1 in the Leydig and Sertoli cells (Figure 4, indicated by arrows) of the testis and the epithelial cells of the ductuli efferentes (Figure 4A,B). Weak fluorescence was also observed in the epididymis (Figure 4C). Omitting the first antibody (negative control) revealed no specific immunofluorescence (Figure 4D). Using a chromogenic approach, staining was mostly overt in the membranes of the Leydig cells, but bright areas were also detected in the seminiferous tubules representing Sertoli cells (Figure 4E,F, arrows), thus confirming the fluorogenic data. To evaluate additional male genital tract tissue, prostate tissue was chosen. Although no expression of T-SP1 protein was overt in normal prostate tissue (Figure 5A), in glandular differentiated prostate carcinoma (Gleason score 6) some cytoplasmic staining was detectable (Figure 5B,C,D, arrows). Finally, ovarian cancer tis- sue sections of different morphological types were tested for the expression of T-SP1 applying the chromogenic staining method. Cytoplasmic staining of the T-SP1 protein was detected in groups of ovarian cancer cells with weak membrane staining (Figure 5E-F, arrows).

Expression of T-SP1 isoforms in HEK293 cells To study the subcellular localization of the T-SP1 splice variants, we designed fusion constructs with the fluorescent protein EGFP at the C-terminus of each proT-SP1 splice variant for transfection of HEK293 cells. Western blot analysis carried out with an anti-EGFP antibody revealed proT-SP1/1-EGFP mainly in the membrane part (Figure 6, M1) of the cells and only in small amounts in the soluble fraction (Figure 6, S1). The fusion protein was detected at the predicted molecular mass of $64 \mathrm{kDa}$. In contrast, proT-SP1/2 (55.7 kDa) and proT-SP1/3 (58.6 kDa) were present primarily in the soluble fraction (Figure 6, S2 and S3) in addition to minor quantities in the membrane fraction (Figure 6, M2 and M3). These results suggest membrane anchoring of proSP1/1, but not of proSP1/2 and proSP1/3.

\section{Discussion}

In this study, we describe cloning, recombinant expression, and initial characterization of a new membraneanchored human serine protease-like protein. According to its main occurrence in testis tissue we now named this
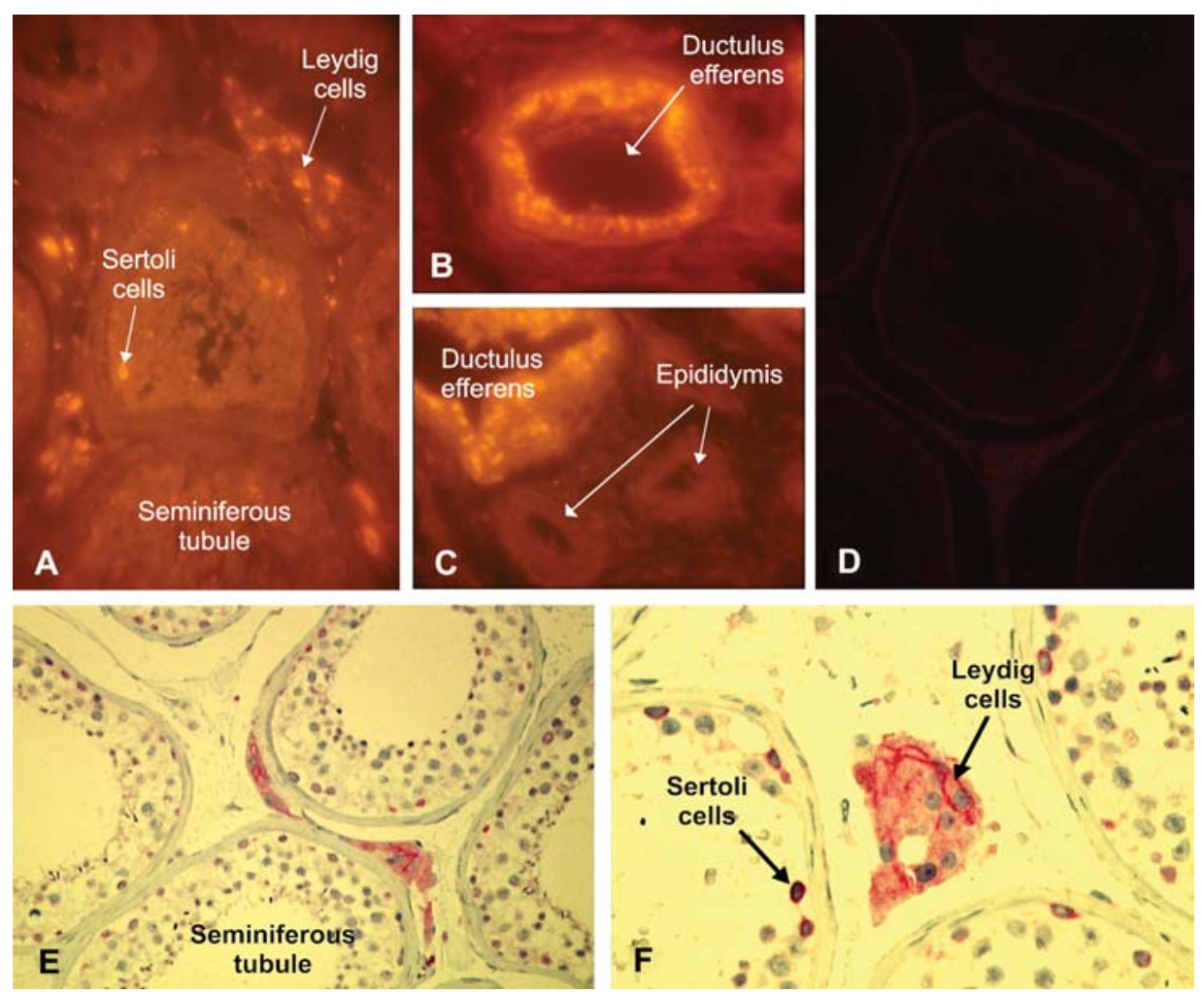

Figure 4 Immunohistochemical analysis of T-SP1 expression in testis tissue.

Immunohistochemical staining of testis tissue was performed using the purified anti-T-SP1 antibody as primary antibody. Intense staining was detected in the Leydig and Sertoli cells of the testis (A, E, and F) and in the epithelial cells of the ductuli efferentes (B and C). Weak staining was observed in the epididymis (C). Omitting the anti-T-SP1 antibody revealed no specific staining (D). Original magnification $1000 \times(A, D, F)$ and $500 \times(B, C, E)$. 

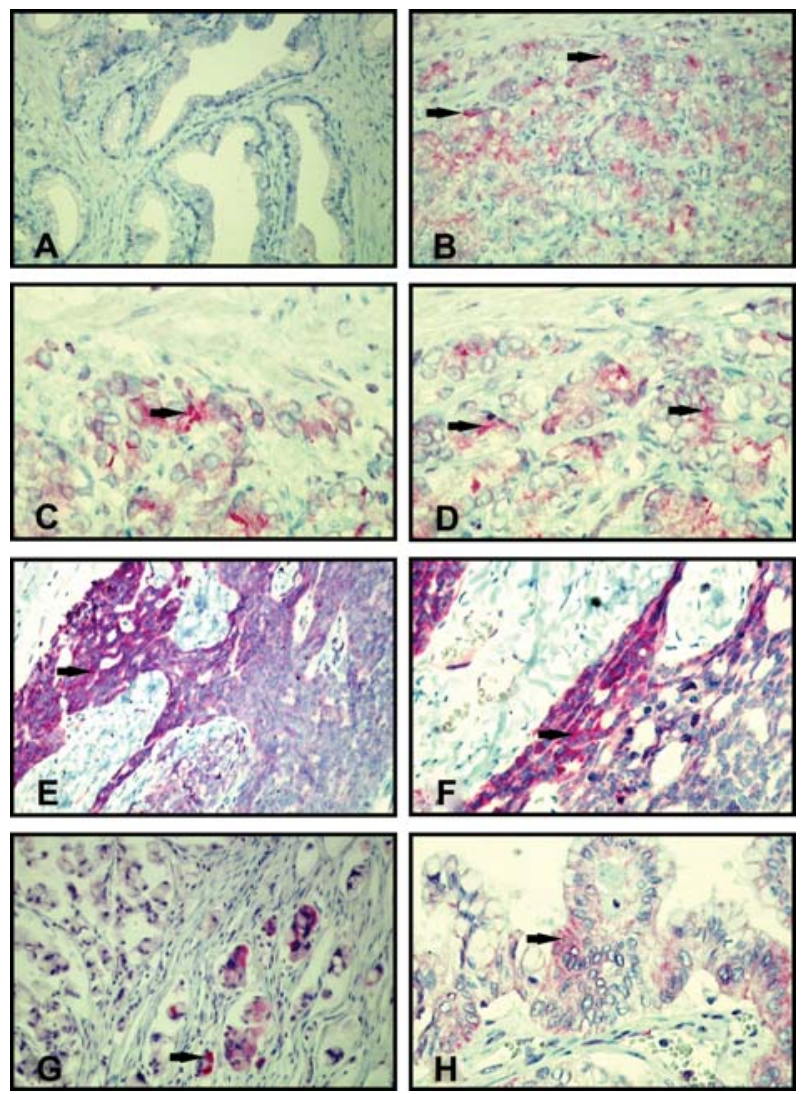

Figure 5 T-SP1 expression in prostate tissue, ovarian cancer tissues, and prostate cancer tissue.

(A) No staining was detected in normal prostate tissue. (B, C, D) Expression of T-SP1 in prostate cancer cells (arrows). (E-H) TSP1 expression was observed in cells of several ovarian cancer tissues (arrows). Original magnification $100 \times(A, E)$ and $200 \times(B$, C, D, F, G, H).

protein testis serine protease 1 (T-SP1). Using our PCRbased assays we were not able to detect T-SP1 in human brain tissue, although 5 EST entries in the UniGene database also suggest expression of this protease-like protein in the brain. However, sequence alignment studies revealed that none of the brain transcripts encode for the full length coding sequence of T-SP1.

The T-SP1 gene is located on the short arm of chromosome 8 and consists of 7 exons comprising approx-

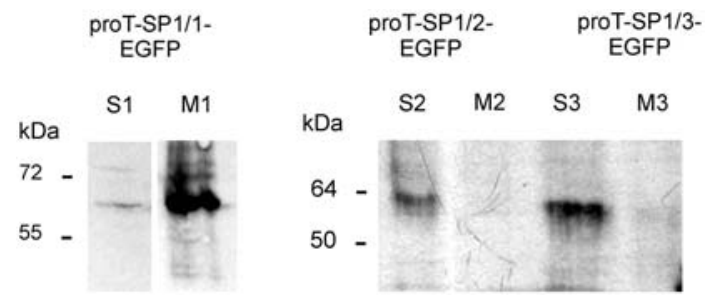

Figure 6 Western blot analysis of recombinantly expressed proT-SP1/1-EGFP forms in HEK293 cells.

ProT-SP1/1-EGFP was mainly detected in the membrane fraction (M1) and only a minor amount was found in the soluble cell fraction (S1), while proT-SP1/2-EGFP and proT-SP1/3-EGFP were mainly detected in the soluble fraction (S2 and S3). For detection of the fusion proteins, a mouse anti-EGFP antibody and a secondary anti-mouse antibody (HRP-linked) were used as described in the materials and methods section. imately $28.7 \mathrm{kbp}$. Apart from the database entry of the mRNA sequences corresponding to variants T-SP1/1 and T-SP1/2, we detected the transcript of a further splice variant in testis tissues which we designated T-SP1/3. The three mRNAs exhibit open reading frames of $1059 \mathrm{bp}$, $831 \mathrm{bp}$, and $912 \mathrm{bp}$, encoding preproproteins with predicted molecular weights of $38.9 \mathrm{kDa}, 30.7 \mathrm{kDa}$, and $33.6 \mathrm{kDa}$, respectively. Immunohistochemical staining with a newly developed polyclonal antibody occurred in the Leydig and Sertoli cells of the testis and in the epithelial cells of the efferent ducts, indicating a potential physiological role of the protein in this male genital tract tissue.

Apart from a reasonable high sequence identity with the coagulation enzymes factor XI (42\%) and plasma kallikrein $(40 \%)$ which arose from a common ancestor by gene duplication (Beaubien et al., 1991), T-SP1/1 also shares some sequence identity with other serine proteases of the male genital tract, such as testisin (36\%), acrosin (35\%), leydin (33\%), and prostasin (35\%). Testisin belongs to the GPI-linked membrane-anchored proteases and is mainly expressed in the testis (Hooper et al., 1999, 2000). Moreover, it is found in some tumor cell lines of other origin (Hooper et al., 2000) and in ovarian tumors (Shigemasa et al., 2000; Tang et al., 2005). For that reason, testisin is supposed to promote malignant transformation in these tumor cells and may, therefore, become a valuable diagnostic marker in certain tumor entities. Moreover, acrosin and leydin are also predominantly expressed in the testis. While acrosin has been studied extensively and is well-characterized as an active protease of the acrosome contributing to the fertilization process (Klemm et al., 1991), leydin is described only once in the literature as a component of the Leydig cells (Poorafshar and Hellman, 1999). Up to now, just cloning and some structural data, but no functional characterization of this testis protein, have been presented.

In contrast to the latter two proteases, which do not belong to the membrane-bound serine proteases, the GPI-anchored prostasin is primarily expressed in the prostate gland and some cancer entities, but not in the testis (Yu et al., 1995; Chen et al., 2001). Yet like testisin, its structure and function is intensively studied (Chen et al., 2004; Shipway et al., 2004) also revealing a prominent role as a potential marker for ovarian cancer (Mok et al., 2001). Interestingly, immunohistochemical staining of T-SP1 with our newly generated polyclonal antibody illustrated a partial membrane localization of the protein in testis tissue. In the cancer sections, T-SP1 revealed a more cytoplasmic staining, indicating a putative intracellular location. At this time, we cannot rule out the possibility that in cancer tissues additional splice variants of T-SP1 were transcribed, possibly without a signal peptide.

Similarly, the human kallikrein-related peptidases, KLK2 and KLK13 (Lundwall et al., 2006), which show a sequence identity of $32 \%$ and $37 \%$, respectively, with the catalytic domain of T-SP1/1, are highly expressed not only in testis but also in ovarian cancer tissues (Tremblay et al., 1996; Petraki et al., 2003; Scorilas et al., 2004; Dorn et al., 2007). Furthermore, KLK5 is upregulated in malignant ovary tissue (Kim et al., 2001), and KLK9 and 
KLK10 have been described as prognosis markers in ovarian cancer (Yousef and Diamandis, 2003). Therefore, it seems possible that T-SP1 may also act as a marker of certain tumors, because we could show its protein expression in human ovarian cancer tissues as well as in glandular differentiated prostate carcinoma, where it may be upregulated by hormones. The latter assumption seems to be underlined by the fact that the MatInspector software (www.genomatix.de) calculates putative binding sites for the progesterone receptor and the androgen receptor 719 bp (TAagtcaTGTTCT) and 1642 bp (GGttCActcTCTTCT) upstream of the transcription initiation site of T-SP1. Thus, T-SP1 expression may be regulated by progesterone and androgens which bind to these receptors (Holdcraft and Braun, 2004; Gadkar-Sable et al., 2005). A hormone-dependent regulation of kallikreinrelated peptidases has already been demonstrated in breast and prostate cancer (Paliouras and Diamandis, 2006, 2008).

With respect to the molecular characterization of the T-SP1 variants, we found a hydrophobic region of 17 amino acids at the C-terminus of T-SP1/1 (WLLLCPLSHVLFRAILY), suggesting that this variant is bound to the plasma membrane. Interestingly, no hydrophobic part at the C-terminus in the sequences of T-SP $1 / 2$ and T-SP1/3 exists. A possible GPI-anchor for T-SP1/1 has already been proposed by other investigators (NetzelArnett et al., 2003). In this review article, T-SP1/1 was classified to belong to a new member of GPI-anchored serine proteases. This anchorage has already been described for several membrane/GPI-bound serine proteases, such as the male genital tract proteases prostasin (Chen et al., 2001) and testisin (Hooper et al., 2000). However, our own studies with phospholipase D treatment of ectopically expressed T-SP1 in HEK293 cells did not result in the release of T-SP1 into the cell culture supernatant (data not shown).

To investigate the role of the different $\mathrm{C}$-termini of the three T-SP1 splice variants, we generated fusion constructs with the fluorescent protein EGFP at the Cterminus of each T-SP1 isoform. Western blot analysis of the ectopically expressed splice variants revealed that the proT-SP1/1-EGFP protein is mainly located in the membrane and only small amounts in the soluble fraction of HEK293 cells. In contrast, the variants proT-SP1/2EGFP and proT-SP1/3-EGFP were primarily detected in the soluble fraction and only in a very small amount in the membrane fraction, suggesting that the C-terminal part of proT-SP1/1 is responsible for membrane anchorage.

For evaluation of the catalytic activity, the purified recombinant T-SP1/1 from E. coli, HEK293 cells, and Pichia pastoris were analyzed. In addition, incubation of the proprotein with furin for zymogen activation was performed, since the recognition sequence (an Arg at positions $\mathrm{P}_{1}, \mathrm{P}_{4}$ and $\mathrm{P}_{6}$ ) (Figure $2 \mathrm{~B}$ ) for cleavage by furin is present in the proregion of proT-SP1/1. Up to now, none of these approaches revealed any proteolytic activity (data not shown).

In summary, we report for the first time the characterization of a new serine protease which is mainly expressed in human testis and, therefore, named by us as testis serine protease 1 (T-SP1). Due to alternative splicing three different variants of T-SP1 exist, but only T-SP1/ 1 may play a physiological role in testis. We describe for the first time the production of recombinant T-SP1 variants and the generation of polyclonal antibodies for immunohistochemical detection. Thus, we demonstrated the presence of T-SP1 in the Leydig and Sertoli cells of the testis and in the efferent ducts, where it might be involved in male fertility, as it has been recently described for KLK14 (Emami et al., 2008). Additionally, under pathological conditions T-SP1 is expressed in prostate cancer and several ovarian cancer tissues and may therefore serve as a novel marker of certain tumor entities.

\section{Materials and methods}

\section{Bioinformatics for the analysis of the T-SP1 gene and protein structure}

The exon-intron structure of the human T-SP1 gene was analyzed by comparing the sequence of experimentally identified T-SP1 transcripts with the genomic sequence available through NCBI using the Blast software (www.ncbi.nlm.nih.gov/BLAST). To identify putative polyA consensus sequences, the WebGene software (www.itb.cnr.it/webgene) was employed. EST entries for T-SP1 transcripts were analyzed using the EST Profile Viewer software (www.ncbi.nlm.nih.gov). Splice variants of T-SP1 were analyzed using the Aceview software (www.ncbi.nlm.nih.gov/ IEB/Research/Acembly). Amino acid and nucleic acid sequence alignments were carried out with the SECentral software (Sci Ed Central, Clone Manager Professional Suite 7.11, Cary, NC, USA).

\section{Cloning of human T-SP1}

The nucleotide sequence (NM_198464), encoding a putative serine protease, was extracted from the GenBank database during an alignment study with human plasma prekallikrein. Based on this nucleotide sequence, primers were designed for cloning of the human T-SP1 cDNA (forward: 5'-CCA TGC TCC TGT TCT CAG TGT TG-3', reverse: 5'-TCA GTA CAA AAT AGC TCT GAA CAA CAC ATG GG-3'). As a template, the human multiple tissue cDNA panels I and II (Clontech, Mountain View, CA, USA) were used. PCR was performed with $5 \mu \mathrm{l}(1 \mathrm{ng}) \mathrm{cDNA}$ as template, $1 \mu \mathrm{l}$ forward and reverse primer (200 nM), $1 \mu \mathrm{l}$ dNTP mix (200 $\mu \mathrm{M}$ each), 10× Hifi buffer (Invitrogen, Carlsbad, CA, USA), $\mathrm{MgSO}_{4}(2 \mathrm{~mm}), 34.5 \mu \mathrm{l} \mathrm{H}_{2} \mathrm{O}$, and $0.5 \mu \mathrm{l}(2.5 \mathrm{U})$ Platinum Taq Polymerase (Invitrogen) in a final volume of $50 \mu \mathrm{l}$. For thermocycling, initial denaturation was carried out at $95^{\circ} \mathrm{C}$ for $2 \mathrm{~min}$ and amplification was achieved by 35 cycles at $95^{\circ} \mathrm{C}$ for $15 \mathrm{~s}$, $58^{\circ} \mathrm{C}$ for $30 \mathrm{~s}$, and $72^{\circ} \mathrm{C}$ for $90 \mathrm{~s}$, followed by a final single incubation step at $72^{\circ} \mathrm{C}$ for $10 \mathrm{~min}$. Cloning of the PCR products was performed using the pCR4-TOPO cloning kit (Invitrogen) according to the protocol of the manufacturer, and the DNA sequence of the clones was determined by a commercial service (MediGenomix, Munich, Germany).

\section{5'-RLM-RACE and 3'-RACE}

Transcriptional start sites of T-SP1 were determined by performing RNA ligase-mediated rapid amplification of 5'-cDNA ends (Gene Racer Kit, Invitrogen) with 250 ng poly $(A)^{+}$mRNA from pooled human testis of 45 males in the age range of 14 to 64 years (Clontech). 5'-RLM-RACE ensures that only full-length 5 '-ends of mRNA molecules with an intact cap structure are selectively reverse transcribed and amplified by nested PCR. For reverse transcription, random oligo-dT priming was employed. 
The T-SP1 specific primers for determination of the transcriptional start sites used in primary and nested PCR were 5'-CCA GTC CAT GAT GAC CAT TG-3' and 5'-AGG ATG GAG CCG CCA CAG AA-3', whereas the primers 5'-CGA CTG GAG CAC GAG GAC ACT GA-3'and 5'-GGA CAC TGA CAT GGA CTG AAG GAG TA-3' served as anchor oligonucleotides. To perform 3'-RACE, the oligonucleotides $5^{\prime}$-GGA TTC TCA CTG CGG CTC AC-3' and 5'-GTG CCG GAT ACA AGA ATG AG-3' were used as genespecific primers, while 5'-GCT GTC AAC GAT ACG CTA CGT AAC G-3' and 5'-CGC TAC GTA ACG GCA TGA CAG TG-3' posed as adapter primers. First and nested PCR were carried out at $95^{\circ} \mathrm{C}$ for $2 \mathrm{~min}$ and amplification was achieved by 35 cycles at $95^{\circ} \mathrm{C}$ for $15 \mathrm{~s}, 52^{\circ} \mathrm{C}$ for $30 \mathrm{~s}$, and $72^{\circ} \mathrm{C}$ for $90 \mathrm{~s}$, followed by a final single incubation step at $72^{\circ} \mathrm{C}$ for $10 \mathrm{~min}$. The PCR products were cloned into the plasmids PCR2.1-TOPO (Invitrogen) and the DNA sequences of the inserts determined as described above.

\section{Detection of T-SP1 transcript expression in human tissue}

Quantitative RT-PCR was carried out using a LightCycler (Roche, Mannheim, Germany) and a LightCycler-FastStart DNA Master SYBR Green I Kit (Roche). Primer sets for the simultaneous as well as separate quantification of all T-SP1 splice variants were designed by Search LC (Heidelberg, Germany). PCR was performed with $1 \mu \mathrm{l} \mathrm{CDNA}(1 \mathrm{ng}$ ) of the human multiple tissue cDNA panels I and II (Clontech) as template, $2 \mu$ primer set (Search LC), $2 \mu$ I LC-FastStart DNA Master SYBR Green I mix, and $15 \mu$ l $\mathrm{H}_{2} \mathrm{O}$. Thermocycling was carried out as follows: initial denaturation was carried out at $95^{\circ} \mathrm{C}$ for $10 \mathrm{~min}$ and amplification was achieved by 45 cycles at $95^{\circ} \mathrm{C}$ for $10 \mathrm{~s}$, at $68^{\circ} \mathrm{C}$ for $10 \mathrm{~s}$, and at $72^{\circ} \mathrm{C}$ for $16 \mathrm{~s}$. Specificity of quantitative PCRs was verified by melting curve analysis and agarose gel electrophoresis.

\section{Expression of recombinant proT-SP1 variants in E. coli and protein purification of proT-SP1}

Clones of the three variants of proT-SP1 in the PCR2.1-TOPO vector as described in 5'-RLM-RACE and $3^{\prime}$-RACE were used as templates. Primers for proT-SP1 were $5^{\prime}$-AAG CCG GCC CAC GGA CTC CTC TCC CAG-3' as forward and 5'-CCG CTC GAG TIT TGC AGA TGA AAG GTG TG-3' as reverse primer for proTSP1/1 and 5'-CGG CCT CGA GTA CAA AAT AGC TCT GAA CAA C- $3^{\prime}$ as reverse primer for proT-SP1/2 and proT-SP1/3, respectively. PCR was performed using the following conditions: denaturation occurred at $95^{\circ} \mathrm{C}$ for $2 \mathrm{~min}$ and amplification was achieved at $95^{\circ} \mathrm{C}$ for $15 \mathrm{~s}, 56^{\circ} \mathrm{C}$ for $30 \mathrm{~s}$ and $72^{\circ} \mathrm{C}$ for $90 \mathrm{~s}$ for 35 cycles, and final extension was at $72^{\circ} \mathrm{C}$ for $10 \mathrm{~min}$. The PCR product was digested with Nael and Xhol and ligated into the pENTR11 vector (Invitrogen). For recombinase reaction, $300 \mathrm{ng}$ pENTR11 vector with insert was incubated with $300 \mathrm{ng}$ pETDEST42 vector and a LR-clonase-mix (Invitrogen) at $25^{\circ} \mathrm{C}$ for $1 \mathrm{~h}$ according to the manufacturer's protocol. After a digestion with proteinase $\mathrm{K}$ at $37^{\circ} \mathrm{C}$ for $10 \mathrm{~min}$, the mixture was used to transform the $E$. coli strain TOP 10 (Invitrogen). Selection was carried out with $200 \mu \mathrm{g} / \mathrm{ml}$ ampicillin and colonies with the correct insert were sequenced by Sequiserve, Munich, Germany. Plasmids with correct in-frame sequences were selected for further studies. The E. coli strain BL21(DE3) was transformed with the respective plasmids for expression of recombinant proT-SP1/1, $-\mathrm{SP} 1 / 2$ and $-\mathrm{SP} 1 / 3$. Expression was induced with $0.5 \mathrm{~mm}$ isopropyl-thio- $\beta$-D-galactoside (IPTG) at an $\mathrm{OD}_{600}$ of 0.4 at $37^{\circ} \mathrm{C}$ and $E$. coli cells were harvested $3 \mathrm{~h}$ after induction. Inclusion bodies were isolated with BugBuster reagent (Novagen, Darmstadt, Germany) according to the instructions of the manufacturer. Briefly, inclusion bodies were dissolved in loading buffer (8 M urea, $100 \mathrm{~mm} \mathrm{NaH}_{2} \mathrm{PO}_{4}, 10 \mathrm{~mm}$ Tris- $\mathrm{HCl}, \mathrm{pH}$ 8) and applied on a 1-ml HisTrap nickel column using the ÄKTAexplorer system (GE Healthcare, Waukesha, WI, USA). After washing with $5 \times$ col-

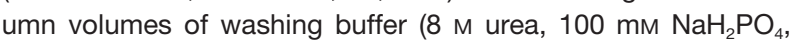
$10 \mathrm{~mm}$ Tris- $\mathrm{HCl}, \mathrm{pH}$ 6.3) recombinant protein was eluted with elution buffer ( $8 \mathrm{M}$ urea, $100 \mathrm{~mm} \mathrm{NaH} \mathrm{PO}_{4}, 10 \mathrm{~mm}$ Tris-HCl, $\mathrm{pH}$ 5.9). Absorbance was monitored at the wavelength at $280 \mathrm{~nm}$. Aliquots of eluted fractions were subjected to SDS-PAGE and stained with Coomassie Blue and assayed for $\mathrm{His}_{6}$-tag immunoreactivity. Recombinant protein was analyzed by amino acid sequencing performed with a gas-phase sequencer (Procice 492 cLC, Applied Biosystems, Darmstadt, Germany) using standard conditions.

\section{Production of polyclonal anti-T-SP1-antibodies}

The purified recombinant T-SP1/3 was used as an antigen to immunize rabbits. Protein $(60 \mu \mathrm{g})$ was injected intramuscularly in two New Zealand white female rabbits. T-SP1/3 was diluted $1: 1$ in complete Freund's adjuvant for the first injection and in incomplete Freund's adjuvant for the following injections. Boosts were carried out 3 times every 3-4 weeks and blood was drawn from the animals and tested for antibody generation in an indirect ELISA.

Anti-T-SP1 antibodies were purified with DEAE Fast Flow Sepharose (GE Healthcare) using the Bio Pilot system (GE Healthcare). The DEAE column was equilibrated with $20 \mathrm{~mm}$ Tris, $\mathrm{pH} 8.5$ and rabbit serum was dialyzed in the same buffer and applied to the column. Elution of the IgGs was carried out using a sodium chloride gradient ( $20 \mathrm{~mm}$ Tris, $\mathrm{pH} 8.5,1 \mathrm{M} \mathrm{NaCl}$ ). IgG fractions were analyzed via SDS-PAGE and Coomassie Blue staining.

\section{Immunohistochemistry}

Immunohistochemical staining of paraffin embedded testis tissue slides (Biocat, Heidelberg, Germany) was performed according to the manufacturer's instructions. Briefly, the primary anti-T-SP1/3 antibody was used in a dilution of $1 / 60$ and incubated overnight at $4^{\circ} \mathrm{C}$. The secondary anti-rabbit-lgG antibody (Santa Cruz Biotechnology Inc., Santa Cruz, CA, USA) was labeled with rhodamine and used in a dilution of 1/100. The slides were embedded with Prolong Antifade (Molecular Probes, Eugene, OR, USA) and analyzed using a fluorescent microscope (IX50 Olympus, Hamburg, Germany). Immunohistochemical staining of testis and diverse cancer tissues with a chromogenic substrate was performed with the AP Polymer System (Zytomed, Berlin, Germany) according to the instruction of the manufacturer with a dilution of the anti-T-SP1/3 antibody of $1 / 150$.

\section{Expression of recombinant T-SP1/1 in HEK293 cells}

EGFP fusion constructs of proT-SP1 variants with the fluorescent protein EGFP at the C-terminus were obtained with a fulllength cDNA cloned into the PCR2.1-TOPO vector as a template. The preproprotein of T-SP1 with its hydrophobic Cterminus was created by PCR with the forward oligonucleotide 5'-GGA ATT CAT GCT CCT GTT CTC AGT GTT GCT-3' with an EcoRI restriction site at the $5^{\prime}$-end and the reverse primer $5^{\prime}$ CGG GAT CCG TAC AAA ATA GCT CTG AAC AAC ACA TGG$3^{\prime}$ or $5^{\prime}$-CGG GAT CCG TIT TGC AGA TGA AAG GTG TGA GAC-3' with a BamHI restriction site at the $3^{\prime}$-end. PCR conditions were as follows: denaturation occurred at $95^{\circ} \mathrm{C}$ for 2 min and amplification was achieved at $95^{\circ} \mathrm{C}$ for $15 \mathrm{~s}, 56^{\circ} \mathrm{C}$ for $30 \mathrm{~s}$ and $72^{\circ} \mathrm{C}$ for $90 \mathrm{~s}$ for 35 cycles and final elongation was at $72^{\circ} \mathrm{C}$ for $10 \mathrm{~min}$. The PCR product was digested with EcoRI and BamHI and ligated into the equally restricted pEGFP-N3 vector (Clontech). 
For transfection with the pEGFP-N3-T-SP1 constructs, HEK 293 cells were plated in a 6-well dish at a density of $30-50 \%$ per well containing $2 \mathrm{ml}$ Dulbecco's modified Eagle's medium (PAA, Pasching, Austria) with $10 \%$ fetal bovine serum and 40 $\mathrm{U} / \mathrm{ml}$ penicillin/streptomycin. The cells were transfected by exposure to $2 \mu \mathrm{g}$ plasmid DNA plus $3 \mu \mathrm{l}$ lipofectamine 2000 (Invitrogen) according to the manufacturer's protocol. After 1 day, cells were split into $10-\mathrm{cm}$ dishes and cultured for 14 days in the presence of $1 \mathrm{mg} / \mathrm{ml}$ G418 (PAA) to select stable transfected cells. Surviving cells were pooled and further expanded in $75-\mathrm{cm}^{2}$ flasks under the selection of $100 \mu \mathrm{g} / \mathrm{ml} \mathrm{G} 418$.

\section{Preparation of soluble and membrane protein fractions in proT-SP1-EGFP expressing HEK293}

For the preparation of membrane and cytosolic fractions of HEK293 cells, confluent cells from a 6-well plate were scraped and centrifuged at $2000 \mathrm{~g}$ for $15 \mathrm{~min}$ at room temperature. The cell pellet was resuspended in lysis buffer $\left(50 \mathrm{~mm} \mathrm{NaH} \mathrm{PO}_{4}\right.$, $50 \mathrm{~mm} \mathrm{Na} \mathrm{HPO}_{4}, 200 \mathrm{~mm} \mathrm{NaCl}, 5 \mathrm{~mm}$ EDTA, 1\% Triton-X 100, $\mathrm{pH}$ 6) with a protease inhibitor cocktail III (Calbiochem/Merck, Darmstadt, Germany) and sonified 3 times for $2 \mathrm{~s}$. Followed by a centrifugation step at $2000 \mathrm{~g}$ for $15 \mathrm{~min}$ at $4^{\circ} \mathrm{C}$, the supernatant (cytosolic fraction) was collected for further analysis. The remaining pellet (membrane fraction) was resuspended in RIPA buffer $(50 \mathrm{~mm}$ Tris-Cl, pH 8, $150 \mathrm{~mm} \mathrm{NaCl}, 1 \% \mathrm{NP}-40,0.5 \%$ sodium deoxycholate and $0.1 \%$ SDS) and rocked on ice for $1 \mathrm{~h}$. Finally, centrifugation was carried out with $10000 \mathrm{~g}$ for $30 \mathrm{~min}$ at $4^{\circ} \mathrm{C}$. The supernatant was further examined by Western blot analysis.

\section{Western blotting of recombinant T-SP1 variants}

Purified proteins were separated under reducing conditions by SDS/PAGE on a $12.5 \%$ gel or on a gradient gel $(4-15 \%$; BioRad, Hercules, CA, USA) and then transferred by electroblotting onto a nitrocellulose membrane (Whatman, Dassel, Germany). The membrane was blocked using blocking buffer [10 mM Tris$\mathrm{Cl} \mathrm{pH} \mathrm{7.5,} 150 \mathrm{~mm} \mathrm{NaCl}, 0.1 \%$ Tween-20 (v/v), 3\% bovine serum albumin (BSA)] for $1 \mathrm{~h}$ at room temperature and incubated with a Penta-His-Conjugate (Qiagen, Hilden, Germany) against the $\mathrm{His}_{6}$-tag linked with HRP (Qiagen) in a dilution of 1:3000 for $1 \mathrm{~h}$ at room temperature. Visualization was achieved by the enhanced chemiluminescence system ECL Plus (GE Healthcare) according to the instructions of the supplier.

For additional detection of T-SP1 the generated polyclonal antibody was used. The blot was blocked with blocking buffer (10 mM Tris-Cl pH 7.5, $150 \mathrm{~mm} \mathrm{NaCl}, 3 \% \mathrm{BSA}$ ) for $1 \mathrm{~h}$ at room temperature and incubated with purified anti-T-SP1/3 antibodies in a dilution of $1: 500$ overnight at $4^{\circ} \mathrm{C}$. As a secondary antibody, the goat anti-rabbit IgG antibody linked with horseradish peroxidase (Dako, Glostrup, Denmark) was used in a dilution of 1:20 000. Visualization was carried out as described above. For the detection of T-SP1-EGFP fusion proteins, an anti-GFP antibody (Roche) was used in a dilution of 1:1000. A secondary HRP-linked anti-mouse antibody (Santa Cruz Biotechnology Inc.) was applied in a dilution of 1:3000.

\section{Acknowledgments}

We thank Reinhard Mentele of the Max-Planck-Institute, Martinsried (Germany) for performing the amino acid sequencing and Prof. Hans Fritz, Prof. Edwin Fink, Dr. Amaury Fernandez Montavan, Dr. Irmgard Machleidt, and Prof. Werner Machleidt for helpful discussions and advice. We thank Prof. Wolf-Bernhard Schill for the evaluation of the immunohistochemical stain- ings. Many thanks also to PD Dr. Alexander Faussner for providing the HEK293 TRex Flp In cells.

\section{References}

Beaubien, G., Rosinski-Chupin, I., Mattei, M.G., Mbikay, M., Chretien, M., and Seidah, N.G. (1991). Gene structure and chromosomal localization of plasma kallikrein. Biochemistry 30, 1628-1635.

Chen, L.M., Skinner, M.L., Kauffman, S.W., Chao, J., Chao, L., Thaler, C.D., and Chai, K.X. (2001). Prostasin is a glycosylphosphatidylinositol-anchored active serine protease. J. Biol. Chem. 276, 21434-21442.

Chen, L.M., Zhang, X., and Chai, K.X. (2004). Regulation of prostasin expression and function in the prostate. Prostate 59, $1-12$.

Clements, J., Hooper, J., Dong, Y., and Harvey, T. (2001). The expanded human kallikrein (KLK) gene family: genomic organisation, tissue-specific expression and potential functions. Biol. Chem. 382, 5-14.

Dorn, J., Schmitt, M., Kates, R., Schmalfeldt, B., Kiechle, M., Scorilas, A., Diamandis, E.P., and Harbeck, N. (2007). Primary tumor levels of human tissue kallikreins affect surgical success and survival in ovarian cancer patients. Clin. Cancer Res. 13, 1742-1748.

Emami, N., Deperthes, D., Malm, J., and Diamandis, E.P. (2008). Major role of the human kallikrein-related peptidase 14 (KLK14) in seminal clot liquefaction. J. Biol. Chem. Epub ahead of print, DOI: 10.1074/jbc.M801194200.

Fink, E., Bhoola, K.D., Snyman, C., Neth, P., and Figueroa, C.D. (2007). Cellular expression of plasma prekallikrein in human tissues. Biol. Chem. 388, 957-963.

Foekens, J.A., Ries, Ch., Look, M.P., Gippner-Steppert, C., Klijn, J.G., and Jochum, M. (2003). Elevated expression of polymorphonuclear leukocyte elastase in breast cancer tissue is associated with tamoxifen failure in patients with advanced disease. Br. J. Cancer 88, 1084-1090.

Gadkar-Sable, S., Shah, C., Rosario, G., Sachdeva, G., and Puri, C. (2005). Progesterone receptors: various forms and functions in reproductive tissues. Front. Biosci. 10, 2118-2130.

Holdcraft, R.W. and Braun, R.E. (2004). Hormonal regulation of spermatogenesis. Int. J. Androl. 27, 335-342.

Hooper, J.D., Nicol, D.L., Dickinson, J.L., Eyre, H.J., Scarman, A.L., Normyle, J.F., Stuttgen, M.A., Douglas, M.L., Loveland, K.A., Sutherland, G.R., et al. (1999). Testisin, a new human serine proteinase expressed by premeiotic testicular germ cells and lost in testicular germ cell tumors. Cancer Res. 59, 3199-3205.

Hooper, J.D., Bowen, N., Marshall, H., Cullen, L.M., Sood, R., Daniels, R., Stuttgen, M.A., Normyle, J.F., Higgs, D.R., Kastner, D.L., et al. (2000). Localization, expression and genomic structure of the gene encoding the human serine protease testisin. Biochim. Biophys. Acta 1492, 63-71.

Jochum, M., Gippner-Steppert, C., Machleidt, W., and Fritz, H. (1994). The role of phagocyte proteinases and proteinase inhibitors in multiple organ failure. Am. J. Respir. Crit. Care Med. 150, S123-S130.

Johnsen, M., Lund, L.R., Romer, J., Almholt, K., and Dano, K. (1998). Cancer invasion and tissue remodeling: common themes in proteolytic matrix degradation. Curr. Opin. Cell Biol. 10, 667-671.

Kim, H., Scorilas, A., Katsaros, D., Yousef, G.M., Massobrio, M., Fracchioli, S., Piccinno, R., Gordini, G., and Diamandis, E.P. (2001). Human kallikrein gene 5 (KLK5) expression is an indicator of poor prognosis in ovarian cancer. Br. J. Cancer 84 , 643-650.

Klemm, U., Muller-Esterl, W., and Engel, W. (1991). Acrosin, the peculiar sperm-specific serine protease. Hum. Genet. 87, 635-641. 
Lilja, H., Ulmert, D., and Vickers, A.J. (2008). Prostate-specific antigen and prostate cancer: prediction, detection and monitoring. Nat. Rev. Cancer 8, 268-278.

Lundwall, A. and Brattsand, M. (2008). Kallikrein-related peptidases. Cell Mol. Life Sci. 65, 2019-2038.

Lundwall, A., Band, V., Blaber, M., Clements, J.A., Courty, Y., Diamandis, E.P., Fritz, H., Lilja, H., Malm, J., Maltais, L.J., et al. (2006). A comprehensive nomenclature for serine proteases with homology to tissue kallikreins. Biol. Chem. 387, 637-641.

McMullen, B.A., Fujikawa, K., and Davie, E.W. (1991a). Location of the disulfide bonds in human coagulation factor XI: the presence of tandem apple domains. Biochemistry 30 , 2056-2060.

McMullen, B.A., Fujikawa, K., and Davie, E.W. (1991b). Location of the disulfide bonds in human plasma prekallikrein: the presence of four novel apple domains in the amino-terminal portion of the molecule. Biochemistry 30, 2050-2056.

Mok, S.C., Chao, J., Skates, S., Wong, K., Yiu, G.K., Muto, M.G., Berkowitz, R.S., and Cramer, D.W. (2001). Prostasin, a potential serum marker for ovarian cancer: identification through microarray technology. J. Natl. Cancer Inst. 93, 1458-1464.

Neth, P., Arnhold, M., Nitschko, H., and Fink, E. (2001). The mRNAs of prekallikrein, factors $\mathrm{XI}$ and XII, and kininogen, components of the contact phase cascade are differentially expressed in multiple non-hepatic human tissues. Thromb. Haemost. 85, 1043-1047.

Neth, P., Arnhold, M., Sidarovich, V., Bhoola, K.D., and Fink, E. (2005). Expression of the plasma prekallikrein gene: utilization of multiple transcription start sites and alternative promoter regions. Biol. Chem. 386, 101-109.

Netzel-Arnett, S., Hooper, J.D., Szabo, R., Madison, E.L., Quigley, J.P., Bugge, T.H., and Antalis, T.M. (2003). Membrane anchored serine proteases: a rapidly expanding group of cell surface proteolytic enzymes with potential roles in cancer. Cancer Metastasis Rev. 22, 237-258.

Paliouras, M. and Diamandis, E.P. (2006). The kallikrein world: an update on the human tissue kallikreins. Biol. Chem. 387, 643-652.

Paliouras, M. and Diamandis, E.P. (2008). Intracellular signaling pathways regulate hormone-dependent kallikrein gene expression. Tumour Biol. 29, 63-75.

Petraki, C.D., Karavana, V.N., and Diamandis, E.P. (2003). Human kallikrein 13 expression in normal tissues: an immunohistochemical study. J. Histochem. Cytochem. 51, 493501.

Poorafshar, M. and Hellman, L. (1999). Cloning and structural analysis of leydin, a novel human serine protease expressed by the Leydig cells of the testis. Eur. J. Biochem. 261, 244-250.

Reuning, U., Sperl, S., Kopitz, C., Kessler, H., Kruger, A., Schmitt, M., and Magdolen, V. (2003). Urokinase-type plasminogen activator (UPA) and its receptor (UPAR): development of antagonists of UPA/UPAR interaction and their effects in vitro and in vivo. Curr. Pharm. Des. 9, 1529-1543.

Scorilas, A., Borgono, C.A., Harbeck, N., Dorn, J., Schmalfeldt, B., Schmitt, M., and Diamandis, E.P. (2004). Human kallikrein 13 protein in ovarian cancer cytosols: a new favorable prognostic marker. J. Clin. Oncol. 22, 678-685.

Shigemasa, K., Underwood, L.J., Beard, J., Tanimoto, H., Ohama, K., Parmley, T.H., and O'Brien, T.J. (2000). Overexpression of testisin, a serine protease expressed by testicular germ cells, in epithelial ovarian tumor cells. J. Soc. Gynecol. Invest. 7, 358-362.

Shipway, A., Danahay, H., Williams, J.A., Tully, D.C., Backes, B.J., and Harris, J.L. (2004). Biochemical characterization of prostasin, a channel activating protease. Biochem. Biophys. Res. Commun. 324, 953-963.

Tang, T., Kmet, M., Corral, L., Vartanian, S., Tobler, A., and Papkoff, J. (2005). Testisin, a glycosyl-phosphatidylinositol-linked serine protease, promotes malignant transformation in vitro and in vivo. Cancer Res. 65, 868-878.

Tremblay, R.R., Deperthes, D., Mailloux, J., Lemay, M., and Dube, J.Y. (1996). Kallikrein expression in a mature cystic ovarian teratoma. J. Clin. Lab. Anal. 10, 229-231.

Waydhas, C., Nast-Kolb, D., Trupka, A., Zettl, R., Kick, M., Wiesholler, J., Schweiberer, L., and Jochum, M. (1996). Posttraumatic inflammatory response, secondary operations, and late multiple organ failure. J. Trauma 40, 624-630.

Whitbread, A.K., Veveris-Lowe, T.L., Lawrence, M.G., Nicol, D.L., and Clements, J.A. (2006). The role of kallikrein-related peptidases in prostate cancer: potential involvement in an epithelial to mesenchymal transition. Biol. Chem. 387, 707-714.

Yousef, G.M. and Diamandis, E.P. (2003). An overview of the kallikrein gene families in humans and other species: emerging candidate tumour markers. Clin. Biochem. 36, 443-452.

Yousef, G.M., Chang, A., Scorilas, A., and Diamandis, E.P. (2000). Genomic organization of the human kallikrein gene family on chromosome 19q13.3-q13.4. Biochem. Biophys. Res. Commun. 276, 125-133.

Yu, J.X., Chao, L., and Chao, J. (1995). Molecular cloning, tissue-specific expression, and cellular localization of human prostasin mRNA. J. Biol. Chem. 270, 13483-13489.

Received June 19, 2008; accepted September 3, 2008 